\title{
Enfermedad tiroidea y gestación en el servicio de alto riesgo obstétrico del Hospital San Vicente de Paúl Medellín 1982-1990
}

\author{
Emilio Alberto Restrepo B.; Alejandro Sierra Lebrun*; Luis Javier Castro Naranjo**; Orlando Montoya***
}

RESUMEN: Se realizó un estudio descriptivo, retrospectivo de pacientes embarazadas y con enfermedad tiroidea asociada, que consultaron al Servicio de Alto Riesgo Obstétrico del Hospital Universitario San Vicente de Paúl de Medellín, en el período comprendido entre el $1^{\circ}$ de enero de 1982 y el 31 de diciembre de 1990 . Se incluyeron aquellas a las que fue posible recopilar todos los datos del formato diseñado para tal fin y que además del control de alto riesgo tuvieron su parto en el Hospital Universitario San Vicente de Paúl.

Se seleccionaron 83 pacientes, siendo el $48,2 \%$ hipertiroideas y el $51,8 \%$ hipotiroideas. El $50 \%$ de las pacientes hipertiroideas tenían enfermedad de Graves y el $20 \%$ de las hipotiroideas enfermedad de Hashimoto.

EI antecedente de infertilidad fue tres veces más frecuente entre las hipotiroideas. Los partos intervenidos se presentaron como antecedente del embarazo anterior en el $25 \%$ de las hipertiroideas y sólo en el $\mathbf{1 3 , 8} \%$ de las hipotiroideas.

El 7,2\% de las pacientes terminaron en aborto. Las hipotiroideas presentaron el doble de abortos en el embarazo actual que las hipertiroideas (4 versus 2 ).

El $18,4 \%$ de las pacientes hipertiroideas presentaron meconio en el líquido amniótico y el $37,1 \%$ sufrimiento fetal.

La hipertensión inducida por el embarazo fue dos veces más frecuente en las hipertiroideas que en las hipotiroideas. Se halló una fuerte asociación estadísticamente significativa entre las pacientes con hipertensión arterial crónica asociada a meconio en líquido amniótico (L.A.) (R.R. = 5,92; $P=0,00456)$.

PALABRAS CLAVES: Enfermedad tiroidea, hipertiroidismo, hipotiroidismo, resultado embarazo.

SUMMARY: We make a descriptive and retrospective analysis of the pregnant woman with thiroid disease that consulted to the service of high risk of obstetrics of the Hospital Universitario San Vicente de Paúl de Medellín, in the period between the january 1 , 1982 and the december 31, 1990.

We found 83 patients, the $48,2 \%$ of this have hyperthyroidism (HTD) and the $51,8 \%$ hypothyroidism (htd). The $50 \%$ of the HTD patients have grave's disease and the $20 \%$ of the htd patients have Hashimoto's disease.

The infertility history was 3 times mor frequent between the htd patients. The instrumental deliveries was more frequent in the last pregnancy $(25 \%)$ in the HTD patients and only in the $13,8 \%$ of the htd patients.

The abortion tase was $7,2 \%$ of all patients. The htd patients presented the double of abortions in the present pregnancy that the HTD patients.

The $\mathbf{1 8 , 4 \%}$ of the HTD patients presented meconium in the amniotic liquid and the $\mathbf{3 7 , 1 \%}$ had fetal distress.

The induced pregnancy hypertension was 2 times more frequent in the HTD that in the htd. We found one strong association stadistically significant between the patients with chronic arterial hypertension asociatted and meconium in amniotic liquid $(R . R .=5,92 ; P=0,00456)$.

KEY WORDS: Thiroid disease, hypothyroidism, hyperthyroidism, pregnancy consequence.

\section{Introducción}

Las enfermedades tiroideas en general se presentan con mayor frecuencia en mujeres que en hombres, con una relación de $4: 1$.

\footnotetext{
* Residentes de tercer año de Ginecología y Obstetricia.

** Ginecobstetra, Profesor U. de A.

*** Odontólogo, Especialista en Epidemiología Centro Regional La

Meseta, Servicio Seccional de Salud de Antioquia.
}

Las mujeres en edad reproductiva padecen trastornos autoinmunes tales como la Enfermedad de Graves y la Enfermedad de Hashimoto, que en sus diferentes períodos de evolución se pueden acompañar de hiper o hipotiroidismo. La Enfermedad de Graves es la principal causa de hipertiroidismo durante el embarazo (1-4).

Aunque inicialmente se consideró como normal el crecimiento de la glándula tiroides durante el embarazo debido a un aumento en la vascularización, el aumento de la globulina trasportadora por acción de los estrógenos y 
un aumento relativo en la T3 y T4 totales, estudios más recientes ponen en tela de juicio este crecimiento de la glándula (4-6).

Cuando se investiga el desarrollo sexual anormal, el hirsutismo, las irregularidades menstruales y la infertilidad, la posibilidad de una disfunción tiroidea debe ser siempre considerada $(1,6-7)$.

El hipotiroidismo puede acompañarse de precocidad sexual, galactorrea, cuerpo lúteo insuficiente (C.L.I.), oligomenorreas, disfunciones ovulatorias, hirsutismo, hiperprolactinemia, hemorragia uterina disfuncional (H.U.D.) y más frecuentemente polimenorreas (1).

El promedio de malformaciones congénitas es tres veces más alta en los hijos de madres hipotiroideas no tratadas que en la población general; sin embargo a pesar del tratamiento el número de malformaciones permanece alto (1).

El hipertiroidismo puede acompañarse de pubertad retardada, desórdenes ovulatorios, infertilidad e hiperemesis gravídica. El hipertiroidismo en el embarazo podría ser dañino para el feto. Los mayores riesgos son prematuridad y tirotoxicosis neonatal. También se ha reportado hipotiroidismo congénito $(1,6)$.

La incidencia de toxemia ha sido reportada como alta $(1,4,7-14)$. En 75 casos descritos, la mortalidad por tirotoxicosis neonatal fue del $16 \%$ (1), otros autores han reportado tasas similares (4).

La prevalencia de hipertiroidismo durante el embarazo es de $0,2 \%(4,9-10)$.

El yodo radioactivo está contraindicado tanto para el diagnóstico como para el tratamiento de la tirotoxicosis durante el embarazo, debido a que el tiroides fetal es capaz de concentrar yodo en épocas tan tempranas como las doce semanas de gestación y además es un órgano muy sensible a la irradiación $(1,3-4,15-16)$.

La destrucción de la glándula tiroidea durante la vida fetal por irradiación resulta en un niño atiroideo, con el riesgo de padecer todas las secuelas descritas en el hipotiroidismo congénito no tratado (cretinismo), siendo el retardo mental la más severa consecuencia del hipotiroidismo congénito. El uso indiscriminado de antitiroideos durante el embarazo, se ha asociado con bocio e hipotiroidismo neonatal $(8,17-20)$.

El desarrollo de la glándula tiroides y el funcionamiento del eje hipotálamo, hipófisis, tiroides, ocurre en forma autónoma sin aparente influencia materna. A las once o doce semanas, el tiroides produce yodotirosinas y yodotironinas, la TSH es detectable y a las 16 semanas alcanza los niveles que se encuentran en el embarazo de término. La T4 llega a los niveles del término a las 20 semanas $(1,6,9)$.

La placenta es fácilmente permeable a TRH, inmunoglobulinas $\mathrm{G}$ y drogas usadas para el tratamiento de la enfermedad tiroidea tales como propiltiouracilo (P.T.U.), metimazol, yodo radioactivo, propanolol. La placenta es relativamente impermeable a TSH, T4, T3. Para otros investigadores, inmunoglobulinas $\mathrm{G}$ como el estimulador tiroideo de acción prolongada (LATS) cruzan la placenta $(3,8)$.
Las tioamidas bloquean la síntesis pero no la liberación de hormonas tiroideas, pueden producir agranulocitosis materna y aplasia cutis en el feto (3-4, 9-10).

La ventaja del PTU sobre el metimazol, consiste en que atraviesa menos y más lentamente la placenta y además bloquea la conversión periférica de T4 a T3 que es la hormona de mayor actividad biológica. La posibilidad de agranulocitosis en la madre es de 1 en $300(4,7$, 21-22).

La muerte fetal, el parto prematuro y el aborto espontáneo, pueden ser disminuidas por el uso de drogas antitiroideas (21).

El uso crónico de propanolol durante el embarazo podría llevar a retardo del crecimiento intrauterino (R.C.I.U.) de tipo simétrico $(10,23)$.

Guerrero Urdina y Reyes Leal, dicen textualmente: "En Colombia, área endémica de bocio, nacen veinte hipotiroideos congénitos por cada mil nacimientos, mientras en países que no son áreas de bocio endémico, nace un cretino por cada 4000 a 5000 nacidos vivos" (24). Queremos llamar la atención sobre este aspecto y además considerar el hijo de madre en tratamiento con tioamidas y yodo radioactivo de alto riesgo para desarrollar hipotiroidismo congénito.

\section{Materiales y métodos}

Se efectuó un estudio retrospectivo, tomando como unidad de análisis las historias clínicas del servicio de alto riesgo obstétrico del Hospital Universitario San Vicente de Paúl de Medellín, de pacientes en embarazo que consultaron entre el 1 de enero de 1982 y el 31 de diciembre de 1990 (9 años). Las variables de interés fueron recolectadas en un formulario diseñado y aprobado para tal fin previa estandarización de los investigadores. La información se procesó por métodos electrónicos bajo el programa Epiinfo, versión 3.1. Se realizó un análisis descriptivo con el total de la población en estudio (83 casos) y se conformaron dos grandes grupos: de hipertiroideas y de hipotiroideas para contrastarlas analíticamente, mediante pruebas de asociación y significancia. Las pacientes se consideraron funcionalmente normales por encontrarse controladas con terapia farmacológica. En el análisis se denominó "Parto o embarazo actual" al evento obstétrico que clasificaba la paciente para su disfunción tiroidea y "Parto o embarazo anterior" al antecedente, en los casos que lo hubo.

\section{Resultados}

De las 83 pacientes gestantes con enfermedad tiroidea el $48,2 \%(n=40)$ eran hipertiroides y el $51,8 \%(n=43)$ hipotiroideas, (Tabla 1).

El 85.5\% $(n=71)$ estaban comprendidas entre los $20 y$ los 35 años de edad, y el $14.5 \%$ restante $(n=12)$ se encontraban en el grupo de edad de 36 a 40 años. No se encontraron pacientes con edades inferiores ni superiores al rango descrito. La edad promedio se registró en 29.8 años con una desviación estándar de 5.32. 


\section{Tabla 1 \\ CARACTERISTICAS DE TERMINACION DEL EMBARAZO ACTUAL Y ANTERIOR EN GESTANTES CON ENFERMEDAD TIROIDEA \\ Hospital Universitario San Vicente de Paúl}

$(\mathbf{n}=\mathbf{8 3})$

\begin{tabular}{|c|c|c|c|c|c|c|c|c|c|c|c|c|}
\hline \multirow{3}{*}{ Atributos } & \multicolumn{6}{|c|}{ Embarazo anterior* } & \multicolumn{6}{|c|}{ Embarazo actual } \\
\hline & \multicolumn{2}{|c|}{ Total } & \multicolumn{2}{|c|}{ Hipertiroid. } & \multicolumn{2}{|c|}{ Hipotiroid. } & \multicolumn{2}{|c|}{ Total } & \multicolumn{2}{|c|}{ Hipertiroid. } & \multicolumn{2}{|c|}{ Hipotiroid. } \\
\hline & $\mathbf{n}$ & $\%$ & $\mathbf{n}$ & $\%$ & $\mathbf{n}$ & $\%$ & $\mathbf{n}$ & $\%$ & $\mathbf{n}$ & $\%$ & $\mathbf{n}$ & $\%$ \\
\hline No. de pacientes & $70 / 83$ & 85.2 & $31 / 70$ & 44.3 & $39 / 70$ & 55.7 & 83 & 100 & $40 / 83$ & 48.2 & $43 / 83$ & 51.8 \\
\hline Abortos & $17 / 70$ & 24.3 & $7 / 31$ & 22.6 & $10 / 39$ & 25.6 & $6 / 8$ & 37.2 & $2 / 40$ & 5.0 & $4 / 43$ & 9.3 \\
\hline Partos & $53 / 70$ & 75.7 & $24 / 31$ & 77.4 & $29 / 39$ & 74.4 & $77 / 83$ & 92.8 & $38 / 40$ & 95.0 & $39 / 43$ & 90.7 \\
\hline -Espontáneos & $43 / 53$ & 81.1 & $18 / 24$ & 75.0 & $25 / 29$ & 86.2 & $42 / 77$ & 54.5 & $18 / 38$ & 47.4 & $24 / 39$ & 61.5 \\
\hline -Intervenidos & $10 / 53$ & 18.9 & $6 / 24$ & 25.0 & $4 / 29$ & 13.8 & $35 / 77$ & 45.5 & $20 / 38$ & 52.6 & $15 / 39$ & 38.5 \\
\hline Meconio & & & & & & & $12 / 77$ & 15.6 & $7 / 38$ & 18.4 & $5 / 39$ & 12.8 \\
\hline Sufrim. Fetal & & & & & & & $18 / 77$ & 23.4 & $13 / 38$ & 34.2 & $5 / 39$ & 12.8 \\
\hline
\end{tabular}

* Se excluyen 13 pacientes sin historia anterior de gestación (Primíparas)

Fuente: Historias Clínicas H.U.S.V.P.

Entre la hipertiroideas, el 50\% $(n=20 / 40)$ se clasificaron como enfermedad de Graves y entre las hipotiroideas, el $11.6 \%(n=5 / 43)$ fueron diagnosticadas como enfermedad de Hashimoto.

En 3 casos (3.6\%) se notificó bocio nodular y en (7.2\%), carcinoma de tiroides.

El embarazo actual terminó en aborto en el 7,2\% $(\mathrm{n}=6)$ y el $1,2 \% \quad(\mathrm{n}=1)$ en muerte fetal intermedia, el $13,3 \%(n=11)$ en parto prematuro, el $75,9 \%(n=63)$ en embarazo a término y el $2,4 \%(n=2)$ como posmaduros. (Fig. 1). Se registraron más abortos entre las hipotiroideas (9.3\%), que entre las hipertiroideas (5.0\%) (Fig. 2). Igual tendencia se había observado con respecto a los abortos en la gestación anterior. (Hipertiroideas 22.6\%, hipotiroideas $25.6 \%$ ).

Con relación a los antecedentes ginecoobstétricos, al $10,8 \%(n=9)$ se le había diagnosticado infertilidad, la que fue tres veces más frecuente entre las hipotiroideas $16,3 \%(n=7)$ que entre las hipertiroideas $5 \%(n=2)$.

El $81,8 \%$ de las oligomenorreas que se reportaron fueron en las pacientes hipotiroideas, como antecedente ginecoobstétrico.

En los casos que llegaron hasta trabajo de parto (Fig. 3) fue más frecuente el parto espontáneo entre las hipotiroideas $(61.5 \%)$ y el intervenido entre las hipertiroideas $(52.6 \%)$. Una distribución semejante había ocurrido en el parto anterior.

Cabe aquí resaltar que la totalidad de los partos pretérmino $(n=3)$, ectópico $(n=1)$ y nacido muerto $(n=1)$ - como terminación del último embarazo, los aportaron pacientes con hipertiroidismo-.

La hipertensión inducida por el embarazo fue dos veces más frecuente entre las pacientes hipertiroideas que en las hipotiroideas. Cuando la paciente con enfer- medad tiroidea tenía además H.T.A. crónica, se halló una fuerte asociación que es estadísticamente significativa con meconio en el líquido amniótico (R.R.= 5,92; P= 0,00456). En los casos en que se asocia hipertensión inducida por el embarazo a enfermedad tiroidea, el riesgo de tener un apgar menor de siete al minuto, se incrementó hasta seis veces (R.R. $=6,24)$, aspecto que fue estadísticamente significativo $(P=0.03695)$.

Para las mujeres con enfermedad tiroidea y diabetes como enfermedad asociada se encontró un RR dos veces mayor de obtener un neonato con sufrimiento fetal agudo $(\mathrm{RR}=2.21)$ y apgar deprimido al minuto $(\mathrm{RR}=2.34)$.

El $15.6 \%$ de las gestantes con enfermedad tiroidea presentaron meconio y el $23.4 \%$ sufrimiento fetal (Fig. 4). Ambos eventos fueron más frecuentes entre las hipertiroideas. Estas reportaron un riesgo de sufrimiento fetal de 3 a 6 veces mayor $(R R=3.12)$ que las mujeres hipotiroideas, asociación que fue estadísticamente significativa (Chi cuadrado $=5.45 ; \mathrm{p}=0.0195$ ) .

Una paciente de 28 años de edad fue expuesta al uso inadvertido de I 131 durante la realización de una gamagrafía en la cuarta semana de gestación. El embarazo transcurrió en forma normal, hasta lograr un parto a término, vértice-espontáneo, cuyo producto no manifestó ningún compromiso, ni depresión del apgar y pesó al nacer 3.000 gramos.

No se conocen los resultados de las pruebas de función tiroidea en los recién nacidos del presente estudio.

Llamó la atención la inusual distribución del sexo en los recién nacidos, en los dos grandes grupos de gestantes con enfermedad tiroidea: mientras en las hipotiroideas por cada mujer nacieron dos hombres, entre las hipertiroideas la relación por sexo fue exactamente la contraria. 
Figura 1

TERMINACION DEL EMBARAZO ACTUAL $(n=83)$

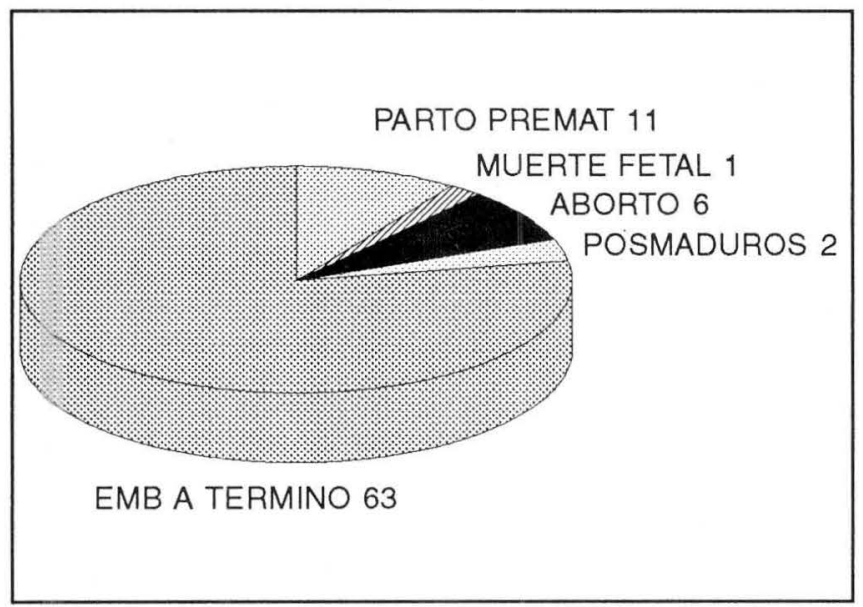

Fuente: Historias Clínicas H.U.S.V.P.

\section{Discusión}

El hipotiroidismo es reconocido como causa de infertilidad, la cual se presentó tres veces más frecuentemente en las pacientes hipotiroideas que en las hipertiroideas en este estudio. El trastorno menstrual predominante fue la oligomenorrea. El $81.9 \%$ de las pacientes que la presentaron eran hipotiroideas.

El hipertiroidismo en general representa un mayor riesgo para la madre y el niño. (Muertes maternas y perinatales, amenaza de parto prematuro, bajo peso al nacer, sufrimiento fetal). En nuestra revisión no encontramos muertes maternas; no hubo tormenta tiroidea, que aunque es una complicación rara, se acompaña de una mortalidad del 15\% (1, 6, 9).

La Enfermedad de Graves resultó ser la primera causa de hipertiroidismo (50\%) como en otros reportes previos $(1,10,15)$. Como enfermedades asociadas al embarazo actual se encontraron la HTA crónica $(9.6 \%)$, diabetes gestacional $(2.4 \%)$ y cardiopatías $(2.4 \%)$. La distribución fue similar tanto en las hipertiroideas como en las
TERMINACION DEL EMBARAZO ACTUAL ENFERMEDAD TIROIDEA Y GESTACION

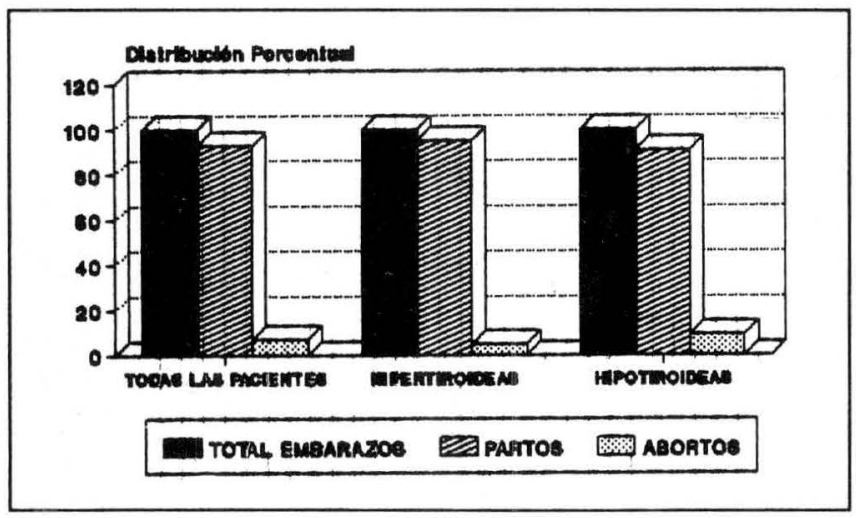

Figura 2

\section{TERMINACION DEL EMBARAZO ANTERIOR ENFERMEDAD TIROIDEA Y GESTACION}

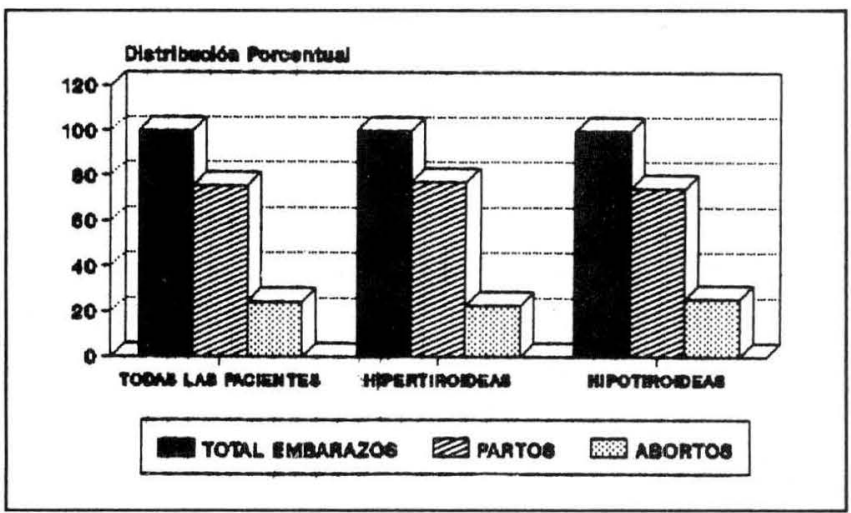

Figura 3

TERMINACION DEL PARTO ACTUAL ENFERMEDAD TIROIDEA Y GESTACION

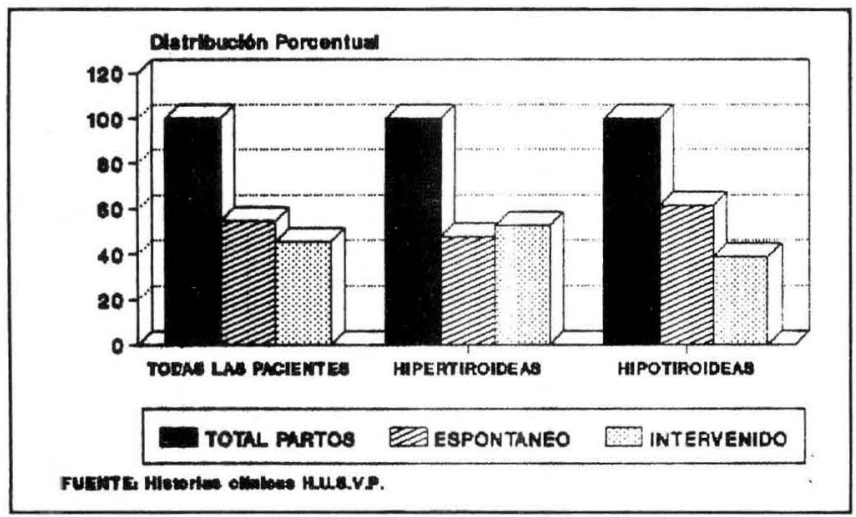

Fuente: Historias Clínicas H.U.S.V.P.
TERMINACION DEL PARTO ANTERIOR ENFERMEDAD TIROIDEA Y GESTACION

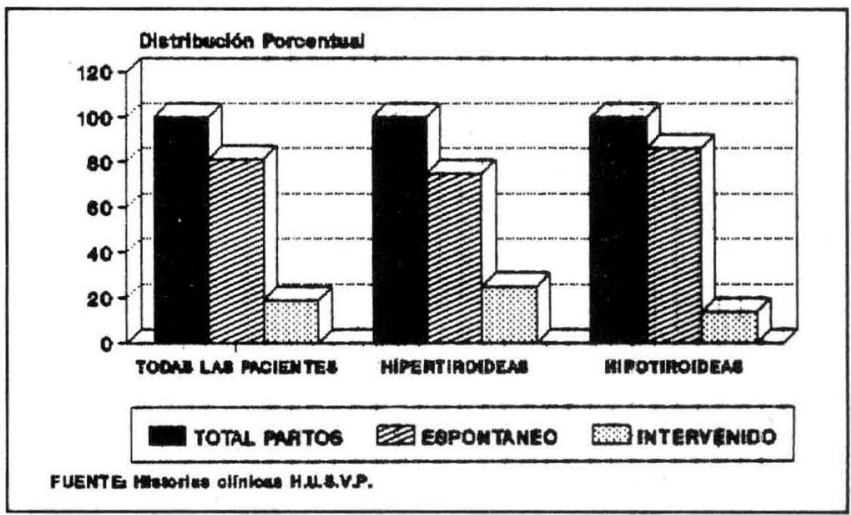

Fuente: Historias Clínicas H.U.S.V.P. 
Figura 4

\section{HALLAZGOS EN EL PARTO ACTUAL ENFERMEDAD TIROIDEA Y GESTACION}

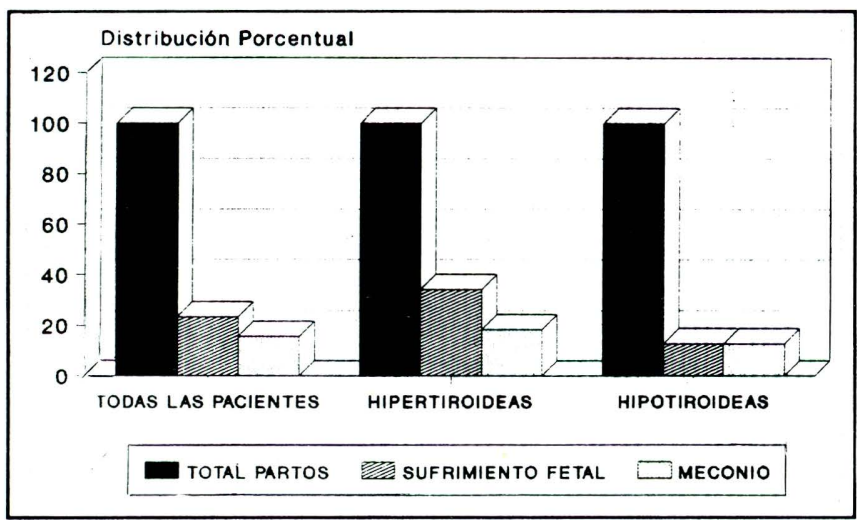

Fuente: Historias Clínicas H.U.S.V.P.

hipotiroideas, excepto para la hipertensión inducida por el embarazo (H.I.E.) que fue dos veces más frecuente entre las primeras que entre las segundas. Esta mayor frecuencia de H.I.E. ha sido demostrada por muchos autores en la paciente hipertiroidea $(2,7,15)$. No hubo asociación con otras variables como la edad materna.
El 7,2\% de los embarazos terminaron en aborto, que está entre la tasa esperada de abortos para la población general.

La frecuencia de aborto fue dos veces mayor en las hipotiroideas, en cambio los partos intervenidos fueron dos veces más frecuentes en las hipertiroideas.

El bajo peso al nacer (menor de 2.500 gramos) en los embarazos de término fue el $11,5 \%$ entre las hipertiroideas $(n=3 / 26)$ y $11,8 \%$ entre las hipotiroideas $(n=4 / 34)$. Un estudio sobre hipotiroidismo subclínico en mujeres embarazadas mostró una tasa de bajo peso al nacer similar de $11.4 \%$ (22).

Con relación al uso de medicamentos no se reportaron reacciones alérgicas al metimazole, droga que se utilizó en el $82,5 \%$ de las pacientes hipertiroideas. No se encontró patología neonatal asociada al uso de propanolol que fue consumido por el $15 \%$ de las pacientes hipertiroideas. En la literatura se relaciona el uso crónico de propanolol con retardo de crecimiento intrauterino (R.C.I.U.) de tipo simétrico $(1,19)$.

En la paciente expuesta al yodo radioactivo parece haber sido crucial para-el buen resultado neonatal, el haber ocurrido en la cuarta semana de gestación, momento en el cual la glándula tiroidea fetal aún no se concentra el yodo $(1,17,21)$.

\section{BIBLIOGRAFIA}

1. Thomas R. and Reid RL. Review. Thyroid disease and reproductive dysfunction: A review; Obstet. Gynecol. 1987; 70: 789-798.

2. Belchetz PE. Thyroid disease in pregnancy. British. Med. J. 1987; 294: 264-265.

3. Gibson M. and Tulchinsky D. The maternal thyroid. Tulchinsky, D. and Ryan K. Maternal fetal endocrinoloy, Saunders, 1980.

4. Becks G. and Burrow GN. Thyroid diseases. The Med. Clin. North. Am. 1991; 75: 121-151.

5. Romano R et al. The effects of Iodoprophylaxis on thyroid size during pregnancy. Am. J. Obstet. Gynecol. 1991; 164: 482-485.

6. Mc Dougall JR. Hyperthyroidism and Maternal Fetal Thyroid Hormone Metabolism. In: Endocrine Disorders in Pregnancy, Editores: Brody S, Ueland K, Kase N, Norwalk, Appleton and Lang. 1989; 151-175.

7. Gómez G., Camacho R. y González L. Enfermedad tiroidea y embarazo. Rev. Col. Obstet. Ginecol. 1991; 42: 125-131.

8. Serup J and Petersen S. Hyperthyroidism during pregnancy treated with propylthiouracil; Acta Obstet. Gynecol. Scand. 1987; 56: 463 466.

9. Burrow GN. The management of thyrotoxicosis in pregnancy: N. Eng. J. Med. 1985; 313: 562.

10. Morales SP. Tiroides y Embarazo. Monografía U. de A. 1987; 63.

11. Chin RR. and Lho TTH. Thyroxine concentration and outcome of hyperemetic pregnancy. British. J. Obstet. Gynecol. 1988; 95: 507509.

12. Page et al. The Pathology of intrauterine thyrotoxicosis: Two cases reports. Obstet. Gynecol. 1988; 72: 479-481.

13. Easterling TR. et al. Maternal hemodynamics in pregnancies complicated by hypertyroidism. Obstet. Gynecol. 1991; 78: 348-352.
14. Mitsud N. et al. Risk factors for develop mental disorders in infants born to women with Grave's disease. Obstet. Gynecol. 1992; 80: 359364.

15. Danon M. Enfermedades del tiroides y embarazo. Cifuentes R. Obstetricia de Alto Riesgo, tercera edición, Cali, Carvajal 1991; 158-251.

16. Noiza $\mathrm{G}$ et al. Early prenatal diagnosis and therapy of fetal hypothyroid goiter. Fetal diagn-ther, 1992; 7(2): 138-143.

17. Morrotanó N., Noh J., Gyanagi H. et al. Antithyroid drug therapy for Grave's Disease during pregnancy: Optim regimen for fetal thyroid Status; Obstet. and Gynecol Survey, 378-379.

18. Peronen F. et al. Women on thyroid hormone therapy. Pregnancy course; fetal outcome and Amniotic fluid thyroid hormone level. Obstet. and Gynecol. 1984; 63: 635.

19. Pruyn SC et al. Long-Term propranol therapy in pregnancy: Maternal and fetal outcome. Am. J. Obstet. Gynecol. 1979; 135: 485-489.

20. Tolivo B de conciliis, B and Mantemagro U. Thyroid hormones in the human pregnancy. Acta Obstet. Gynecol. Scand. 1985; 64: 557-559.

21. Reycraft D. Enfermedad de Graves; Clinic. Obstet. y Ginec. 1983; 3: 745-778.

22. Bruner et al. Diabetes mellitus and Grave's disease in pregnancy complicated by maternal allergies to antithyroid medication. Obstet. Gynecol. 1988; 72: 443-445.

23. Morley et al. Thyrotropin-releasing hormone and Thyroid hormones in amniotic fluid. Am. J. Obstet. Gynecol. 1979; 134: 581-584.

24. Guerrero S. y Reyes B. Hipotiroidismo subclínico en mujeres embarazadas. Rev. Col. Obstet. Ginecol. 1992; 43: 67. 